\title{
Abnormal Biomarkers at Rest in Individuals with Sickle Cell Trait
}

\section{Introduction}

Sickle cell anemia was first reported in 1910 by James B. Herrick, a physician practicing in Chicago, who described a 20-year-old black Caribbean dental student as having shortness of breath, palpitations, yellow eyes, and anemia. ${ }^{1}$ Once the disease was characterized as a single gene mutation, homozygotes were described as having sickle cell disease (SCD) while heterozygotes were thought to be benign carriers and given the designation sickle cell trait (SCT). SCD is estimated to affect 100,000 US citizens; 1 in every 365 African-American births and 1 in 16,300 Hispanic-American births. ${ }^{2,3}$ The prevalence of SCT among African-Americans in the US is about $8 \%$ (1 in 13 African-American births) representing 3 million affected Americans and 300 million worldwide. ${ }^{4-6}$ Much research has been done on SCD but in the 1970s and early 1980s observations were published suggesting individuals with SCT might be better classified as having a mild clinical condition. ${ }^{4,7-9}$ After much debate, the conversation resurfaced thirty years later. ${ }^{5,6,10,11}$ The observations suggesting that SCT may be a clinical condition occurred within two primary groups; warfighters and athletes, and in two primary forms; exertion-related sudden death events, and chronic, subclinical, health concerns.

The first report of exercise-related sudden death (ERD) among US warfighters with sickle cell trait was in 1970 at Ft. Bliss, TX when four Army recruits died during basic training. These four recruits were among the $2 \%$ of all recruits that year with sickle cell trait accounting for one death for every 600 recruits with sickle cell trait. Among the five reported warfighters who died from exercise-related events from 1965-1981, four had sickle cell trait while the fifth was a compound heterozygote (HbSC) ${ }^{12,13}$ In 1987, Kark reported that $7.8 \%$ of military recruits had SCT and described the association between strenuous exercise and the risk of sudden death among soldiers with SCT. The risk of non-traumatic sudden death in black recruits was 32-fold higher than non-black recruits and 27.6-fold higher for black recruits with SCT compared to black recruits without SCT. ${ }^{12}$ Among recruits with SCT, the rate of exercise-related death was 11$30 / 100,000$ recruit training cycles compared to $1 / 100,000$ recruit training cycles without SCT. This risk normalized after basic training for three primary reasons: 1 . most new recruits enter basic training without previous physical conditioning putting them at risk; 2 . physical demands lessen after basic training reducing the risk; 3. those who experience exercise-related events discontinue service selecting for those with lower risk. Physiologically, exercise-related events produce hypoxia and acidosis which promotes sickling leading to death primarily from rhabdomyolysis, heat stroke, and cardiac arrythmias. ${ }^{13}$

Sudden death associated with SCT was first reported on the athletic field in 1974 involving an American collegiate football player. ${ }^{13-15}$ Although sudden death has been observed in a variety of sports, football has produced the most reports and garnered the most press. Similar to the military, the prevalence of professional football players with SCT was reported to be $6.7 \%$ in 
1973, consistent with the general population. ${ }^{16}$ NCAA Division I football athletes with SCT are estimated at $3-4 \%{ }^{17}$ and have a relative risk of death that is 37 times greater than those without SCT; this risk is 15 times greater when considering all NCAA sports. ${ }^{18}$ It is estimated that approximately 5300 NCAA athletes across all sports have SCT. ${ }^{17}$ Between 2000-2010 sixteen deaths occurred in NCAA Division I football and all sixteen were during conditioning drills, none were during games. Of these 16 deaths four were classified as cardiac, one was asthma, one was exertional heat stroke and ten $(63 \%)$ were associated with complications of exertional sickling. ${ }^{17}$

In 2012 O'Connor et. al. assembled military, athletic, and hematology experts resulting in a publication describing guidelines to mitigate the risks of exercise collapse associated with sickle cell trait (ECAST) for warfighters and athletes. ${ }^{19}$ Also in 2012 the National Athletic Trainers Association published practice guidelines for the care of athletes with SCT that focused on universal precautions for all athletes along with specific guidelines for those with SCT to include an awareness of symptoms of rhabdomyolysis and sickling for the individual and all those present during conditioning sessions. ${ }^{20}$

In addition to ECAST, a wide variety of health concerns were also reported in individuals with SCT. Clinical complications with a "definite" connection to SCT have been described as; renal medullary carcinoma, hematuria, renal papillary necrosis, hyposthenuria, and splenic infarction. Other clinical issues that have "probable" associations with SCT include; complicated hyphema, venous thromboembolism, fetal loss/demise, and low birth weight. Clinical conditions with a "weak or possible" association with SCT are; acute chest syndrome, asymptomatic bacteremia in pregnancy, and proliferative retinopathy. ${ }^{10}$

Hypercoagulable state is present in patients with $\mathrm{SCT}^{21}$ and the level of activation increases with disease severity from AS to SC to SS. ${ }^{22}$ Thrombotic activation in individuals with SCT contributes to the risk of thromboembolism. ${ }^{23,24}$ Elevated D-dimers, thombin/antithrombin complexes, and fibrinogen fragment 1.2 have been reported in AS subjects at rest confirming baseline thrombotic activity and providing potential biomarkers to identify thrombotic activation. ${ }^{21,25}$

SCT is now recognized as a contributing factor for ECAST and ERD in warfighters and athletes and poses potential health risks for all who bear the mutated gene. Rhabdomyolysis is the primary pathophysiologic event present in ECAST and ERD ${ }^{4-6,9-20,26}$ but thrombosis, ${ }^{21-25}$ sickling, ${ }^{12-20}$ renal dysfunction, ${ }^{27-29}$ inflammation, ${ }^{30-32}$ and oxidative stres ${ }^{33-35}$ have also been reported. However, ECAST and/or ERD are relatively unpredictable events. The concept of the "perfect storm" has been postulated retrospectively whereby activation of one or more of these pathophysiologic pathways in combination with dehydration, exercise intensity, diet, and a history of rhabdomyolysis contribute to ECAST, though the relationship of these factors is imperfect. ${ }^{36}$ It would be ideal if the risk of a 'perfect storm' could be forecasted for an individual prior to participation. 
Since thrombosis activation has been documented in SCT subjects at rest and it increases with disease severity, it is possible that subclinical activation might predict risk of ECAST. Similarly, if blood or urine biomarkers for myolysis, hemolysis from sickling, renal dysfunction, or inflammation could be identified that are also elevated at rest, a risk profile for ECAST may be possible. A mechanism to predict risk of ECAST can equip athletic trainers, military supervisors, and overseeing physicians with tools to reduce adverse outcomes and provide equal opportunity for individuals with SCT to serve in the military and compete in athletics.

The purposes of this pilot study were to corroborate finding of activated coagulation at rest in SCT subjects, extend findings to biomarkers that assess pathophysiologic pathways other than thrombosis, and evaluate variability of abnormal baseline biomarkers over time.

\section{Materials and Methods}

\section{Research Subjects:}

Four subjects with SCT and four healthy subjects without sickle cell trait were IRB consented and enrolled in the study. All subjects were between the ages of 18 and 60 years and no ongoing health issues were self-reported.

\section{Sample Collection:}

Blood and urine samples were collected from each subject on four different occasions at least two weeks apart. At the first collection session three tubes of blood were collected (EDTA, red top tube, sodium citrate) using standard venipuncture techniques along with one random urine sample. For collection sessions \#2, \#3, and \#4, two blood tubes were collected (red top tubes and sodium citrate) with a random urine sample. Urine testing was performed within two hours of collection and blood samples were processed and serum/plasma samples were stored at $-18^{0} \mathrm{C}$ for future batch testing.

\section{Sample Testing:}

The EDTA blood samples from the first collection were used to verify HbS genotype (AA or AS) using a hemoglobin solubility screen (Sickle Confirm [in-house method]) and hemoglobin electrophoresis confirmation (Quick Gel [Helena Lab, Beaumont, TX).

Plasma from the sodium citrate tube was used to test for D-dimer (latex agglutination [Remel ThermoFisher Scientific, Waltham, MA]) and fibrin monomer (hemeagglutination [Diagnostica Stago, Parsippany, NJ]).

Serum samples were tested for total protein (dye binding) and creatine kinase (enzymatic method) using the Ace Alera Clinical Chemistry Analyzer (Alfa Wassermann, West Caldwell, NJ). Haptoglobin was measured by radial immunodiffusion (Kent Laboratories, Bellingham, WA). 
A chemical urinalysis (dipstick) and a microalbumin (dipstick) were performed on each sample using the CLIAWaived Autourinalysis Reagent test strips and the CLIAWaived microalbumin 21 combo strips, respectively.

\section{Results:}

Mean values for the serum analytes; creatine kinase (CK), total protein (TP), and haptoglobin were compared between the HbAS (SCT) group and HbAA (normal control) group. Each collection time point was treated independently creating 16 data points for each group. Mean values were plotted with $95 \%$ confidence intervals and statistically analyzed using an independent T-test with the alpha set at 0.05 .

The AS group produced higher serum CK levels compared to the control group but the difference was not statistically significant $(\mathrm{p}=0.25)$. The $95 \%$ confidence intervals suggest a greater variability in CK levels in the AS group compared to the AA group (Figure 1).

The AS group produced higher serum TP levels compared to the control group but the difference was not statistically significant $(\mathrm{p}=0.27)$. The $95 \%$ confidence intervals show similar variability between the AS and AA groups (Figure 2).

As can be seen in Figure 3, the AS group produced lower serum haptoglobin levels compared to the control group and the difference was statistically significant $(p=0.006)$. The $95 \%$ confidence intervals show similar variability in the AS and AA groups.

D-dimer and fibrin monomer were performed on citrated plasma from the 16 samples in each group. Results were recorded as positive or negative and samples positive for D-dimer were diluted and retested to estimate concentration.

Three of four AS subjects had at least one positive D-dimer results with one AS subject showing elevated D-dimers at all four time points (Table 1). Overall, 9 of 16 (56.3\%) AS blood samples produced a positive D-dimer result. Two of four AS subjects produced a positive fibrin monomer result, one positive result each among the four time points, totaling $2 / 16$ positive samples (12.5\%). Interestingly, the AS subject with normal D-dimer levels at all four time points produced a positive fibrin monomer result. The other AS subject with a positive fibrin monomer had a positive D-dimer in all four samples collected. All AA subjects in the control group had negative (normal) D-dimer and fibrin monomer results at all four time points.

Samples with positive D-dimer results were diluted to produce a semiquantitative concentration for each analyte. Table 2 shows the estimated levels of D-dimer for each positive result. D-dimer levels in positive AS samples ranged from 1-2 ug/mL to $4-8 \mathrm{ug} / \mathrm{mL}$.

Between sample variability was observed for both the D-dimer and fibrin monomer levels because some AS subjects were positive and some were negative. Likewise, within-sample 
variability was also observed as some time points produced a positive result for the D-dimer or fibrin monomer test and other time points were negative.

A urine sample was collected on each subject in both groups at all four time points and a chemical urinalysis (dipstick) was performed along with a microalbumin analysis. No abnormal results were obtained for any subject, at any time interval, in either group with one exception. One AS subject showed a positive urine leukocyte from a slightly cloudy urine and self-reported symptoms of a mild urinary tract infection.

\section{Discussion:}

The primary aims of this pilot study were to: confirm that some subjects with SCT will show evidence of subclinical thrombotic activity by virtue of elevated D-dimer and/or fibrin monomer levels at rest; and to test other blood and urine biomarkers for similar baseline abnormalities. In addition to testing for subclinical thrombotic activity, we were also interested in the possibility of subclinical myolysis, hemolysis, and renal dysfunction. We sought to screen for subclinical myolysis activity by detecting elevated creatine kinase in the blood and elevated blood and/or protein (both due to myoglobin) in the urine. Subclinical hemolysis was evaluated in blood samples by measuring a decrease in haptoglobin and in the urine by detecting elevated blood and/or protein (both due to hemoglobin). Renal dysfunction was evaluated by detecting any type of abnormality in the chemical urinalysis with an emphasis in blood, protein, and specific gravity on the dipstick.

Although not statistically significant, $\mathrm{CK}$ and total protein levels were higher in the AS population compared to the AA control group. CK is an enzyme found in various tissues but is highest in muscle and brain. One specific isotype, CK-2 (CK-MB), is highest in skeletal muscle tissue and is released upon skeletal muscle injury. An elevation of CK in the AS group is consistent with subclinical myolysis. Similarly, during bouts of intravascular hemolysis hemoglobin is released from erythrocytes into the blood. Both $\mathrm{CK}$ and hemoglobin, if released in sufficient amounts from myocytes and erythrocytes respectively, could produce an elevated total protein as seen in the AS group. It is unclear if the statistically insignificant difference between groups is clinically insignificant and explained by random variability or if a larger sample size might produce statistically significant differences.

A statistically significant difference in blood haptoglobin levels between groups was observed. During episodes of intravascular hemolysis, the hemoglobin released from erythrocytes will bind to haptoglobin to be transported to the liver for detoxification and excretion. Once at the liver haptoglobin releases the hemoglobin which is converted to bilirubin, delivered to the gall bladder as a component of bile, and eventually excreted in the feces as urobilinogen. Lower blood haptoglobin levels in the AS group suggest subclinical intravascular hemolysis. 
Elevated D-dimers and fibrin monomers corroborate previously reported thrombotic activity at rest in subjects with $\mathrm{SCT}^{25}$ and showed two important findings related to subclinical thrombotic activation. First, the frequency of D-dimer (56.3\%) and fibrin monomer (12.5\%) elevations suggest that thrombotic activation is a viable marker to study further with potential to predict or identify ECAST. Second, the within-subject variability of positive results suggests that risk of ECAST may change day-to-day in AS subjects, presumably in response to external factors that influence thrombotic activation. This variability may require ongoing screening prior to each exercise event by those caring for athletes and warfighters prone to ECAST. AS subject \#4, a black female in her 50s, had elevated D-dimer levels at all four blood samplings. Interestingly, AS subject \#1, also a black female in her 50s, demonstrated positive D-dimer results at three of four blood samplings. In contrast, AS subject \#3, a black female in her 30s, showed two positive D-dimer results while AS subject \#2, a black female in her 20s, had no elevated D-dimer levels at any of the four samplings.

Funding and subject recruitment limitations prohibited assessment of a full range of potential biomarkers. Several biomarkers, other than those chosen in this study, are available to measure myolysis (myoglobin), thrombotic activation (thrombin/antithrombin complexes, fibrinogen fragment 1.2, Protein C, Protein S), hemolysis (plasma hemoglobin, reticulocytes), renal dysfunction (BUN, creatinine, creatinine clearance), inflammation (CRP, hsCRP, ferritin), and oxidative stress (advanced oxidation protein products, malondialdehyde, nitrotyrosine, ferricreducing antioxidant power, nitrite, and nitrate).

Future studies will include assessing: 1. a wider selection of biomarkers on subjects with SCT at rest to determine those most sensitive to subclinical pathophysiologic activations; 2. biomarker levels after moderate exercise to identify "responders" who show greater post-exercise elevations compared to baseline; 3 . biomarker levels over time to verify within and between-subject variability. Assessing abnormal biomarkers at rest will further inform subclinical pathophysiology and expand biomarkers that may be useful in clinical practice. Biomarkers that elevate following exercise presumes greater risk for ECAST and identifies biomarkers to potentially predict risk and identify "responders" to exercise. If exercise responders also show baseline biomarker elevations, resting measurements of these biomarkers may be useful to predict responders. An ability to predict responders will equip health care providers with tools to counsel individuals about risk, customize work out routines to minimize risk, and identify early stages of ECAST which together may reduce ECAST and ERD.

Table 1. D-dimer and fibrin monomer results for the AS group (SCT) using an agglutination method. Results were reported as the number positive results per total tests performed. All samples tested in the AA (normal control) group were negative at all four collection time points.

Positive D-dimer and Fibrin Monomer Results 


\begin{tabular}{|l|l|l|l|l|l|}
\hline Test & Subject 1 & Subject 2 & Subject 3 & Subject 4 & Total Positive \\
\hline D-dimer & 3/4 positive & 0/4 positive & 2/4 positive & $4 / 4$ positive & $9 / 16=56.3 \%$ \\
\hline Fibrin Monomer & 0/4 positive & 1/4 positive & 0/4 positive & $1 / 4$ positive & $2 / 16=12.5 \%$ \\
\hline
\end{tabular}

* All D-dimer and fibrin monomer tests were negative in all four AA subjects at all four time points.

Table 2. Estimated concentration of D-dimer in positive AS samples. Concentration is estimated based on the highest dilution that produced agglutination.

Estimated Concentration of D-dimer in Positive AS Samples

\begin{tabular}{|l|l|l|l|l|l|}
\hline $\begin{array}{c}\text { Subject } \\
\text { Number }\end{array}$ & $\begin{array}{c}\text { Sample } \\
\text { Number }\end{array}$ & \multicolumn{1}{|c|}{$\begin{array}{c}\text { No } \\
\text { Dilution }\end{array}$} & $\begin{array}{c}1: 2 \\
\text { Dilution }\end{array}$ & $\begin{array}{c}\text { 1:4 } \\
\text { Dilution }\end{array}$ & $\begin{array}{c}\text { Estimated } \\
\text { Concentration }\end{array}$ \\
\hline AS Subject \#1 & Sample \#1 & Negative & N/A & N/A & $<1.0 \mathrm{ug} / \mathrm{mL}$ \\
\hline & Sample \#2 & Positive & Positive & Negative & $2-4 \mathrm{ug} / \mathrm{mL}$ \\
\hline & Sample \#3 & Positive & Positive & Negative & $2-4 \mathrm{ug} / \mathrm{mL}$ \\
\hline & Sample \#4 & Positive & Positive & Negative & $2-4 \mathrm{ug} / \mathrm{mL}$ \\
\hline AS Subject \#2 & Sample \#1 & Negative & N/A & N/A & $<1.0 \mathrm{ug} / \mathrm{mL}$ \\
\hline & Sample \#2 & Negative & N/A & N/A & $<1.0 \mathrm{ug} / \mathrm{mL}$ \\
\hline & Sample \#3 & Negative & N/A & N/A & $<1.0 \mathrm{ug} / \mathrm{mL}$ \\
\hline & Sample \#4 & Negative & N/A & N/A & $<1.0 \mathrm{ug} / \mathrm{mL}$ \\
\hline AS Subject \#3 & Sample \#1 & Positive & Positive & Negative & $2-4 \mathrm{ug} / \mathrm{mL}$ \\
\hline & Sample \#2 & Positive & Positive & Negative & $2-4 \mathrm{ug} / \mathrm{mL}$ \\
\hline & Sample \#3 & Negative & N/A & N/A & $<1.0 \mathrm{ug} / \mathrm{mL}$ \\
\hline & Sample \#4 & Negative & N/A & N/A & $<1.0 \mathrm{ug} / \mathrm{mL}$ \\
\hline AS Subject \#4 & Sample \#1 & Positive & Negative & N/A & $1-2 \mathrm{ug} / \mathrm{mL}$ \\
\hline & Sample \#2 & Positive & Positive & Negative & $2-4 \mathrm{ug} / \mathrm{mL}$ \\
\hline & Sample \#3 & Positive & Positive & Positive & $4-8 \mathrm{ug} / \mathrm{mL}$ \\
\hline & Sample \#4 & Positive & Positive & Positive & $4-8 \mathrm{ug} / \mathrm{mL}$ \\
\hline
\end{tabular}


Creatine Kinase (CK)

$$
\text { ( } p=0.25 \text { ) }
$$

140

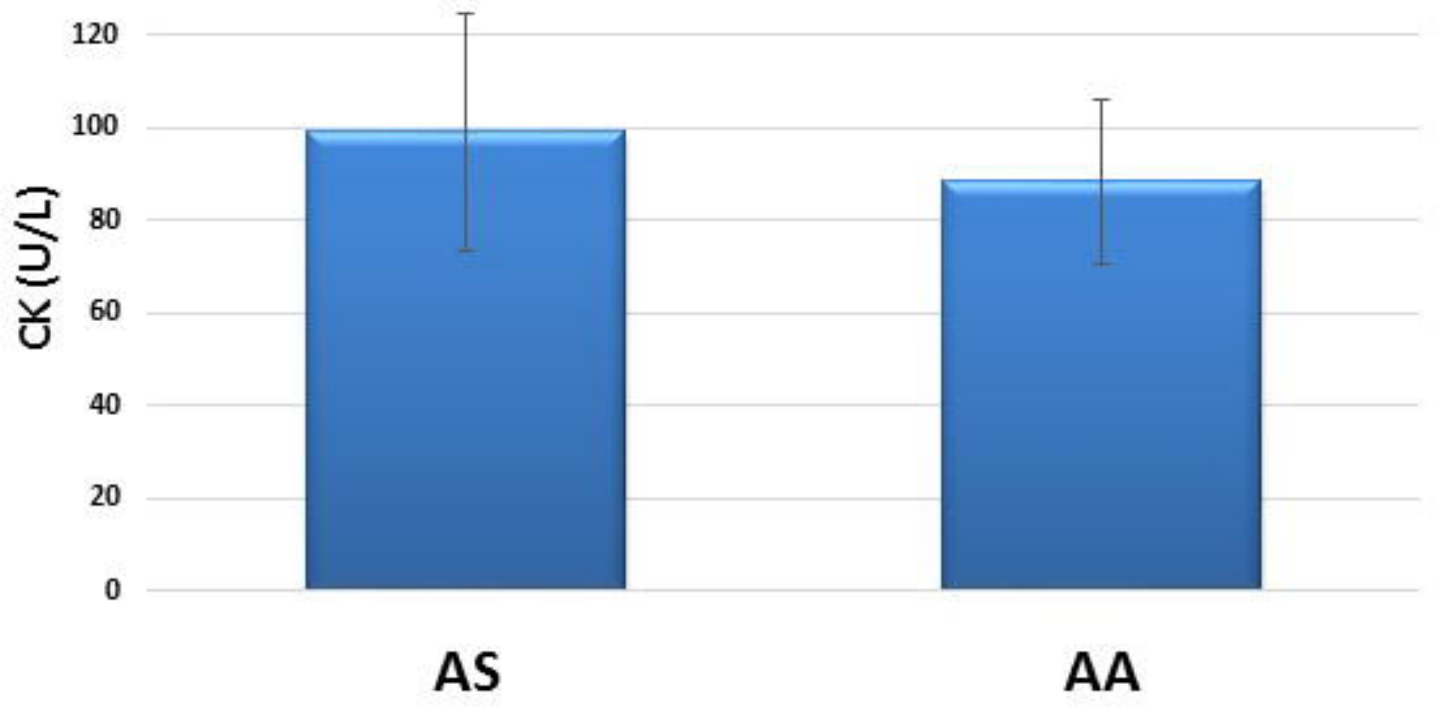


Total Protein (TP)

$$
(p=0.27)
$$

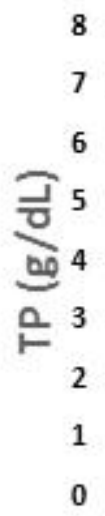

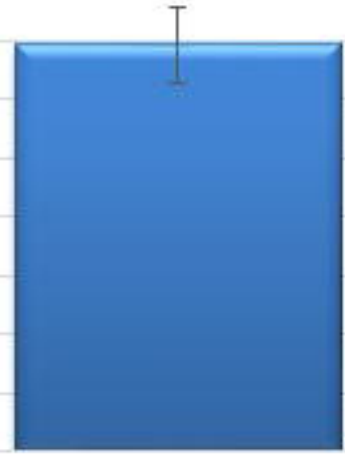

AS

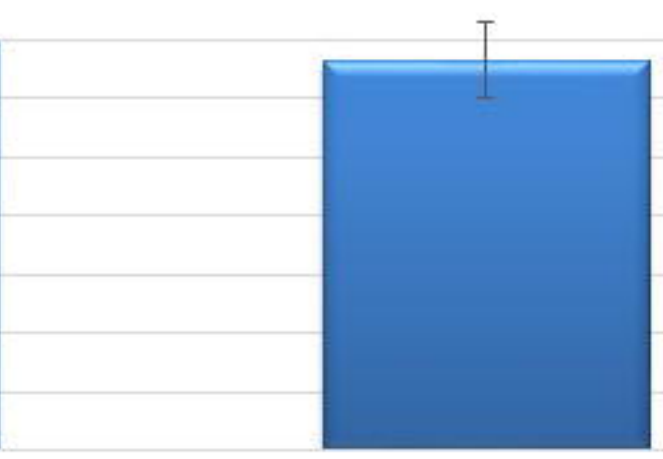

AA 


\section{Haptoglobin}

$$
(p=0.006)
$$

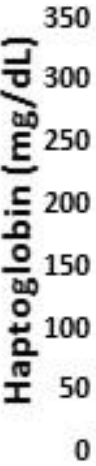

